\title{
Simulation and Experimental Test in Tensile Behaviour of Austenitic Stainless Steels
}

\author{
Pham Quang*, Trinh Huu Toan \\ School of Materials Science and Engineering, Hanoi University of Science and Technology, Hanoi, Vietnam \\ Email address: \\ quang.pham@hust.edu.vn (P. Quang), toan.huutrinh@hust.edu.vn (T. H. Toan) \\ ${ }^{*}$ Corresponding author
}

To cite this article:

Pham Quang, Trinh Huu Toan. Simulation and Experimental Test in Tensile Behaviour of Austenitic Stainless Steels. Advances in Materials. Vol. 8, No. 3, 2019, pp. 108-111. doi: 10.11648/j.am.20190803.12

Received: June 5, 2019; Accepted: July 18, 2019; Published: July 31, 2019

\begin{abstract}
In the systems of fuel cell (FC) and nuclear safety (NS) components many liners of ultra-high pressure tanks and pipes are directly exposed to hydrogen. Austenitic stainless steels are used as material for FC and NS components because of their high resistance to hydrogen intrusion. It is reported that hydrogen degrades mechanical properties of metals significantly. In the hydrogen-charged specimen of SUS 304, a desired model would be able to capture the mechanisms found in experimental testing like large strain elasticity, rate dependence, amplitude dependence, creep and damage. Thus, a prediction of material failure/fracture, including its behavior at large plastic deformations is of importance. To validate existing failure models, the finite element (FE) simulations are used in terms of dependence on length scale and strain state. Restrictions made the selection limited to, in Abaqus, already existing models. Axisymmetric simulations are performed in Abaqus to verify the material model required in order to capture the necking phenomenon in tensile testing. The elasto-plastic modeling in the FE simulations is directed ultimately to initiation and propagation of tension processes. Furthermore, numerical simulation results using the sub-models of crack-tip meshes are discussed. In our experiments, the tensile test system MTS at a crosshead speed of $1 \mathrm{~mm} / \mathrm{s}$ are conducted, which enabled accurate monitoring of displacements on the specimen surfaces. When a material reached the limit of its capacity to carry further loading, deformations localize into necking and became highly dependent on the length over which the strain evaluation is performed the length scale.
\end{abstract}

Keywords: SUS 304 Stainless Steel, Evaluation of Joint Strength, Tensile Strength, FEM

\section{Introduction}

In sheet metal forming processes of fuel cell (FC) and nuclear safety (NS) components many liners of ultra-high pressure tanks and pipes are directly exposed to hydrogen. It has been reported that hydrogen degrades mechanical properties of metals significantly. Modeling of the material failure/fracture, including the behavior at large plastic deformations, is critical for accurate failure predictions.

There have been many studies on fracture and ductility of steels in hydrogen environment under static stress. It is known that reduction of area is intensively reduced in stainless steels subjected to tensile stress in high-pressure hydrogen environment [1]. This is presumed to arise from crack nucleation on the specimen surface by tensile stress and embrittlement at the crack tip [1, 2]. However, Murakami et al. pointed out that the crack having a length longer than a critical value Lc decreases the reduction of area in tensile test $[3,4]$. In addition, in order to ensure safety and reliability of FC and NS systems over the long term, the most important thing is to understand the effects of hydrogen on fatigue behavior. Austenitic stainless steels will be used for materials of FC and NS components because of their high resistance to hydrogen intrusion. However, there have been few systematic studies on effects of hydrogen on fatigue strength in stainless steels. The objective of the present study is to clarify the influences of hydrogen on crack growth and loss of ductility.

The compression model for stainless steel has a significant impact on predicting the structural behavior of stainless steel columns and beams by numerical simulation [5-9]. These constituent models have been verified by comparing their results with test data from the compression tests published in the literature [10-12]. After two decades of development, 
finite element methods have been successfully applied to the analysis of many large deformation elasto-plastic problems. Since the establishment of finite element methods to solve linear problems, an extension to nonlinear elasto-plastic analysis was started by Argyris [13], Pope [14], Marcal and King [15], and Yamada et al. [16, 17]. Stiffness matrices based on the incremental stress-strain relation were derived for small strain problems. Some techniques to control the transition region at the elasto-plastic interface were introduced. Another approach using an 'initial stress' computational process was proposed by Zienkiewicz et al. [18]. The finite element (FE) method is an established numerical tool suitable for these purposes.

In our experiments, the MTS measuring system was used, which enabled accurate monitoring of displacements on the specimen surfaces. When a material reached the limit of its capacity to carry further loading, deformations localize into necking and became highly dependent on the length over which the strain evaluation is performed the length scale.

In FE simulations, this length scale dependence must be taken into account when using elements of different sizes in the mesh. Furthermore, the strain axiality also had an impact on the necking and fracture behavior of the material which should be accounted for in FE simulations. The capacity of some failure criteria available in the FE software Abaqus was investigated. Also, the results from the FE simulations of the tests were presented. Kindly be noted that throughout the current investigation stresses and strains were always the true stresses and strains unless otherwise stated.

\section{Experimental}

The materials used in this study were flat bars made of a type 304 stainless steel (SUS 304). Table 1 shows the chemical compositions of the materials. The tensile tests were conducted. The specimen dimensions follow the norm ISO 527-2/1A (ASTM E8M90a) [19] and are shown in Figure $1(\mathrm{a}, \mathrm{b})$. The current investigation may have an engineering fracture strain of up to $40 \%$, with a speed of 1 $\mathrm{mm} / \mathrm{s}$. In the test, it's allowed for an engineering strain of up to $50 \%$ at the end of the test.

When performing an uniaxial tension test, most polymers begin to deform inhomogeneously at relatively small strains. This process is called necking and usually starts before the softening according to the stress-strain curve. In the initiation of necking a collection of occurs rapidly and generally at the peak load or nominal yield stress. Since the stress state is difficult to define as well as the strain and strain rate vary appreciably over the gauge section the neck complicates measurements of material properties. The neck stabilizes and the deformation continues to propagate with relatively constant force and with some creep-like response within the material. With continuous strain the softening process will lead to a hardening process until failure.

Table 1. Composition of SUS 304 used (mass\%).

\begin{tabular}{llllllll}
\hline $\mathbf{C}$ & $\mathbf{S i}$ & Mn & $\mathbf{P}$ & $\mathbf{S}$ & $\mathbf{N i}$ & $\mathbf{C r}$ & $\mathbf{M e}$ \\
\hline 0.06 & 0.67 & 1.01 & 0.029 & 0.009 & 8.50 & 18 & - \\
\hline
\end{tabular}

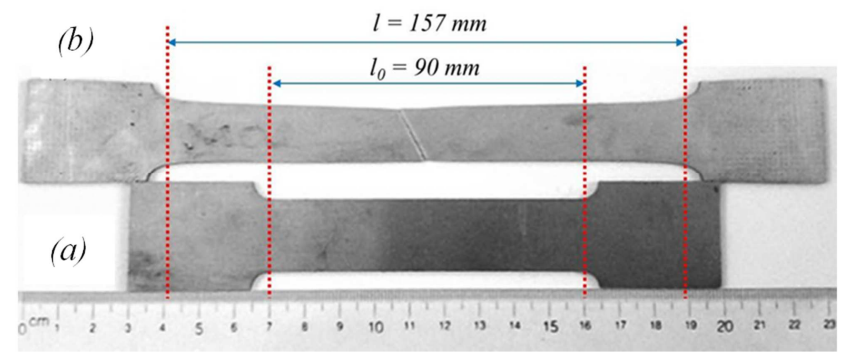

Figure 1. The tensile sample before (a) and after test (b).

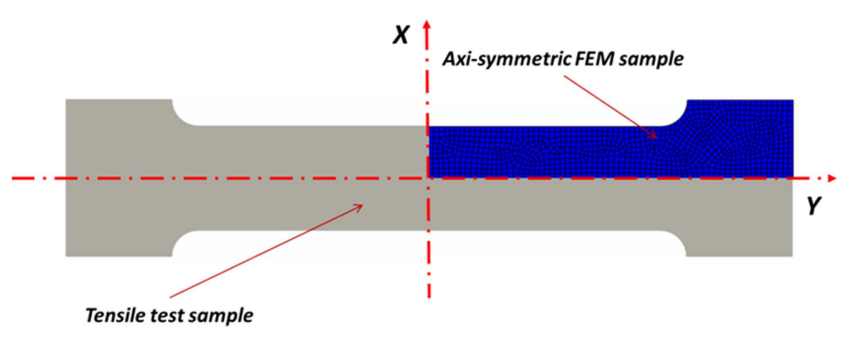

Figure 2. FE axisymmetric model of a simulation workpiece.

\section{Numerical Method}

Many three-dimensional field problems happen in engineering exhibit symmetry about an axis of rotation. Such problems, known as axisymmetric problems, can be solved using two-dimensional finite elements, which are most conveniently described in cylindrical $(r, \theta, z)$ coordinates. The required conditions for a problem to be axisymmetric are as follows: i) The domain problem must possess an axis of symmetry, which is conventionally taken as the $z$ axis; that is, the domain is geometrically a solid of revolution. ii) The boundary conditions are symmetric about the axis of revolution; thus, all boundary conditions are independent of the circumferential coordinate $\theta$. iii) All loading conditions are symmetric about the axis of revolution; thus, they are also independent of the circumferential coordinate. In addition, the material properties must be symmetric about the axis of revolution. This condition is, of course, automatically satisfied for isotropic materials.

Abaqus/CAE [20] embeds the part in the $X-Y$ plane with the $Y$-axis indicating the axis of revolution. An axisymmetric part can contain a combination of only planar shell and wire features, and all cut features are defined as planar through cuts. The model of an axisymmetric part is used to perform this simulation. In the present work, the dimensions of a workpiece are similar to these of MTS test sample shown in Figure 2. Due to axisymmetry, one quarter of the specimen is modeled by 925 numbers of finite elements defined by the used shell elements. 
Abaqus requires these values to interpret the data correctly. Quite often material test data are supplied using values of nominal stress and strain. In such situations the expressions presented below must be used to convert the plastic material data from nominal stress/strain values to true stress/strain values. The relationship between true stress and nominal stress and strain is [21]:

$$
\sigma=\sigma_{\text {nom }}\left(1+\varepsilon_{\text {nom }}\right)
$$

where $\sigma$ is the true strain and $\sigma_{\text {nom }}$ and $\varepsilon_{\text {nom }}$ are the nominal strain and nominal tress.

\section{Results and Discussions}

Figure 3 shows the experimental tensile test curve (a) and the simulation curve (b) at load speed of $1 \mathrm{~mm} / \mathrm{s}$. The good results of simulation are compared with MTS tested sample. These specimens are drawn to approximately about 65\% (67 $\mathrm{mm})$ strain.

Tests performed with lower rates result in more plastic deformation slightly before necking. The curve shown in Figure 3 is a typical illustration of the results that should be obtained von Mises and displacement increments are taken to

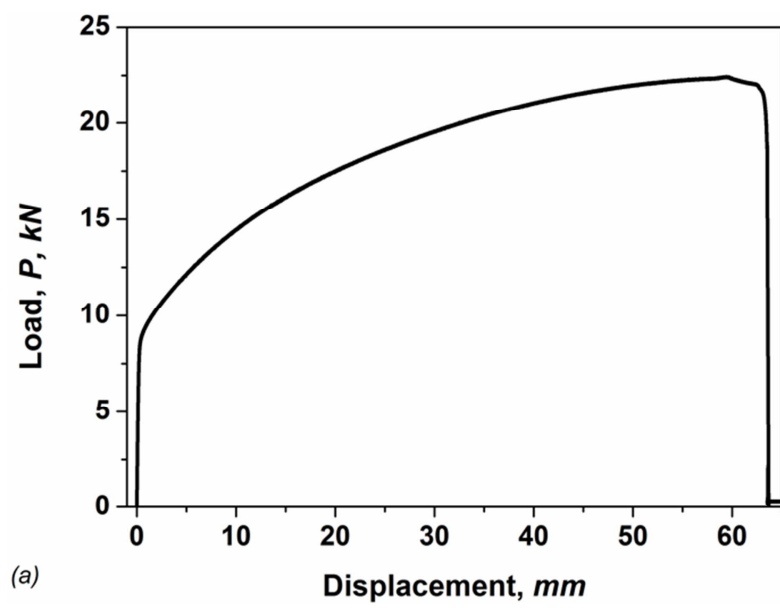

complete this analysis up to $65 \%$ elongation. Figure $4(\mathrm{a}, \mathrm{b})$ shows a workpiece after loading, effective stress is of 817 MPa max. The engineering strain is defined as $100 \%$ and the change in the cross-sectional area is from 3 to $1.5 \mathrm{~mm}$.

After the modification of the elasto-plastic curve, the simulated true stress-strain curve shows good agreement with the target curve during uniaxial static loading. Up to the point of neck initiation, the model shows good agreement with the experiments in the tensile tests. However, the model had difficulties in simulating the necking phenomenon. In real experiments, the initiation of necking is a continuous process where the polymer chains relocate; whereas in the simulations, this process cannot be simulated and the neck forms very rapidly. Even the initiation of necking starts too early.

In the experiments, no sign of a neck was visible after a displacement of $67 \mathrm{~mm}$, whereas Figures 4 (a) and (b) clearly show an initiation of a neck. The fact that the force level is incorrect after loading completion makes the unloading false. The simulations show an overestimation of the remaining displacement after unloading because it starts on a lower force level. After formation of the neck, the model again shows good agreement with the simulation during loading.

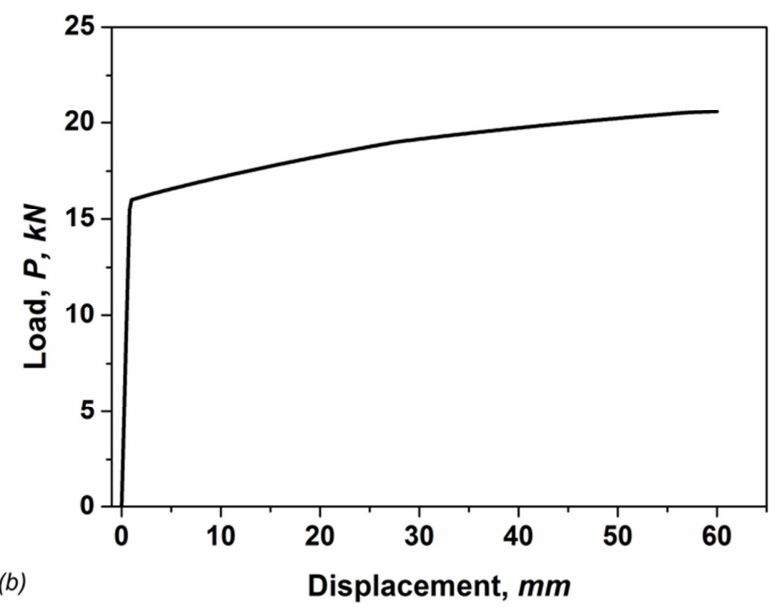

Figure 3. The experimental tensile curve (a) and the simulation one (b).

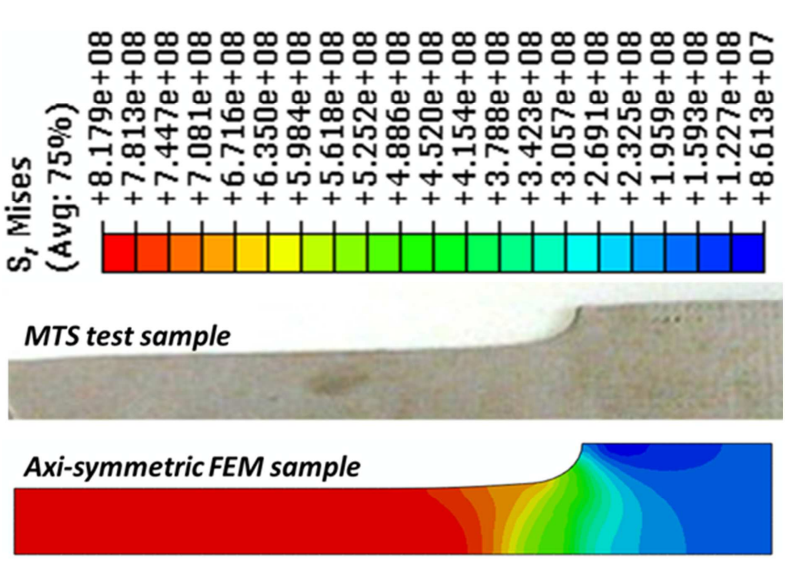

(a)

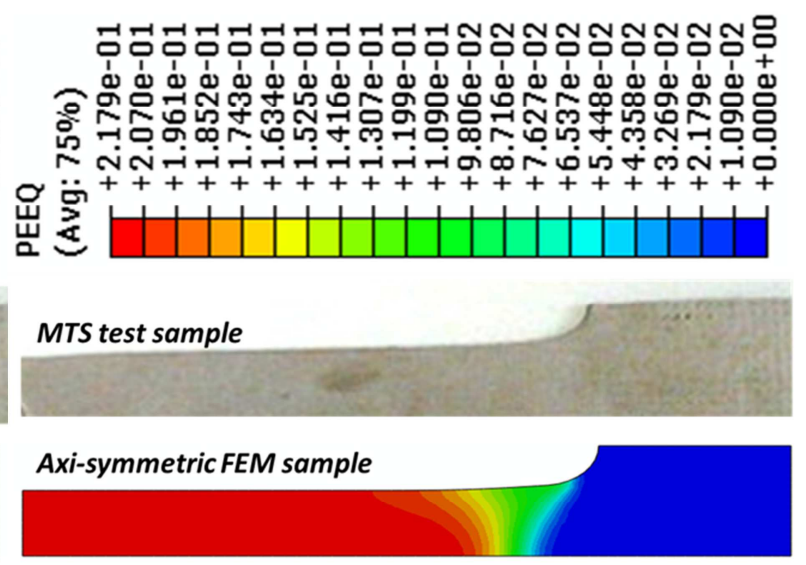

(b)

Figure 4. FE model compared to experimental data for tensile test. 


\section{Conclusions}

In the hydrogen-charged specimen of SUS 304, a desired model would be able to capture the mechanisms found in experimental testing like large strain elasticity, rate dependence, amplitude dependence, creep and damage. Such a model does not exist today. May complicate it even the material appeared to be time dependent both in elasticity and in plasticity. Restrictions made the selection limited to, in Abaqus, already existing models. Axisymmetric simulations are performed in Abaqus to verify the material model to tensile test. Axisymmetric simulations are required in order to capture the necking phenomenon in tensile testing.

The good results of simulation are compared with MTS tested sample. These specimens are drawn to approximately about $65 \%$ (67 $\mathrm{mm})$ strain.

The effective stress is of $817 \mathrm{MPa}$ max. The engineering strain is defined as $100 \%$ and the change in the crosssectional area is from 3 to $1.5 \mathrm{~mm}$.

\section{Acknowledgements}

This study was carried out Technology in framework of the Ministry of Industry and Trade and the Vietnam National Foundation for Science and Technology Development (NAFOSTED) No. 107.01-2018.25.

\section{References}

[1] Jr. R. B. Benson, R. K. Dann and Jr. L. W. Robe rt, Trans. Metall. Soc. AIME, 242, (1968) pp. 2199-2205.

[2] E. Herms, J. M. Olive and M. Puiggali, Mater. Sci. and Eng., A 272 (1999) pp. 279-283.

[3] Y. Murakami, C. Makabe and H. Nisitani, J. Testing and Evaluation, JTEVA, 17 (1989) pp. 20-27.
[4] Y. Murakami. and K. J. Miller, Proceedings of the Cumulative Fatigue Damage Conference, Ed. Navarro A., Seville, Spain (2003).

[5] Z. Tao, B. Uy, F. Y. Liao, L. H. Han. J. Constr. Steel Res. (2011).

[6] M. Patton, K. Singh. Thin-Walled Struct. (2012).

[7] M. Patton, K. Singh. Thin-Walled Struct. (2013).

[8] V. Patel, Q. Q. Liang, M. Hadi. J. Constr. Steel Res. (2014).

[9] V. Patel, Q. Q. Liang, M. Hadi. Eng. Struct. (2017).

[10] L. Gardner, M. Ashraf Eng. Struct. (2006).

[11] W. M. Quach, J. G. Teng, K. F. Chung. J. Struct. Eng. (2008).

[12] L. Gardner, D. Nethercot. J. Constr. Steel Res. (2004).

[13] J. H. Argyris, Proc. First Conf. Matrix Methods in Struct. Mech., AFFDL-TR-66- 80 (1966) pp. 11-190.

[14] G. G. Pope, Aeron. Quart. 17 (1966) pp. 83-104.

[15] P. V. Marcal and I. P. King, Internat. J. Mech. Sci. (1967) pp. 43-155.

[16] Y. Yamada, N. Yoshimura and T. Sakurai, Internat. J. Mech. Sci. 10 (1968) pp. 343-354.

[17] Y. Yamada, T. Kawai, N. Yoshimura and T. Sakurai, Proc. 2nd Conf. on Matrix Methods in Struct. Mech., AFFDL-TR-68150 (1969) pp. 1271-1299.

[18] O. C. Zienkiewicz, S. Valliappan and I. King P., Internat. J. Numer. Meths. Eng. (1969) pp. 75-100.

[19] E. A. Brandes, "Smithells metals reference book", Butterworths, 1983.

[20] Abaqus/CAE, "User's Manual".

[21] Abaqus, Example Problems Manual Volume I: "Static and Dynamic Analyses". 